\title{
Genetic variation and parental performance under inbreeding for growth in Eucalyptus globulus
}

\author{
João Costa E SILVA ${ }^{1,2 *}$, Craig HARDNER ${ }^{3}$, Brad M. PotTs ${ }^{4}$ \\ ${ }^{1}$ Centro de Estudos Florestais, Departamento de Engenharia Florestal, Instituto Superior de Agronomia, Universidade Técnica de Lisboa, \\ Tapada da Ajuda, 1349-017 Lisboa Codex, Portugal \\ ${ }^{2}$ Centro de Matemática e Aplicações, Departamento de Matemática, Instituto Superior Técnico, Universidade Técnica de Lisboa, \\ Avenida Rovisco Pais, 1049-001 Lisboa, Portugal \\ ${ }^{3}$ University of Queensland, School of Land, Crop and Food Sciences, St Lucia 4072 Queensland, Australia \\ ${ }^{4}$ School of Plant Science and Cooperative Research Centre for Forestry, University of Tasmania, Private Bag 55, Hobart, Tas. 7001, Australia
}

(Received 1 June 2009; accepted 21 December 2009)

Keywords:

Eucalyptus globulus / self-fertilization /

mixed mating /

inbreeding depression /

genetic variance /

heritability /

breeding value

\begin{abstract}
- We aimed to better understand the genetic architecture of growth in E. globulus undergoing inbreeding by comparing families from selfing (SELF), open pollination (OP) and unrelated polymix crossing (POL) of common parents. Stem diameter at breast height (DBH) was assessed at 4, 6 and 10 years after planting in a field trial.

- The OP heritability was overestimated at an early age relative to the POL heritability.

- No significant correlations were found between the SELF and POL parental effects, indicating substantial non-additive genetic variation under inbreeding. The OP family effects were better correlated with the SELF than the POL population, and only at age 10 years, after substantial mortality of inbred progeny has occurred, was the positive correlation between OP and POL families significantly different from zero.

- The estimated dominance variance arising from inbreeding was nearly 10 -fold greater than the dominance variance associated with random mating and the additive variance, and appeared to be a major contributor to the variation in inbreeding depression amongst selfed families.
\end{abstract}

\section{INTRODUCTION}

Inbreeding can lead to a reduction in the mean performance of a population, which is usually referred to as inbreeding depression (Lynch and Walsh, 1998). Inbreeding also complicates the genetic covariance structure of a population, introducing additional genetic parameters to express the covariance of relatives when compared with a random mating population (Cockerham, 1983; Harris, 1964). There are few studies comparing the quantitative genetic architecture of inbred and outbred populations (Edwards and Lamkey, 2002; Kelly and Arathi, 2003; Shaw et al., 1998), particularly in long-lived forest trees such as eucalypts (Hardner et al., 1996a; Resende and Vencovsky, 1992). This issue is important as most forest species are in the early stages of domestication, and many breeders are still exploiting open-pollinated seed collections from the wild (Eldridge et al., 1993). Indeed, the relative magnitude of the additive and non-additive genetic effects will be a key determinant of selection efficiency in the early phase of domestication.

A key question in estimating and utilising breeding values from open-pollinated trials is the extent to which variation be-

\footnotetext{
*Corresponding author: jces@isa.utl.pt
}

tween families reflects the additive genetic variation amongst the seed parents (Borralho, 1994). This was initially studied in Eucalyptus globulus by collection of both open-pollinated seed and pollen from the same wild trees, and then crossing these trees as male parents in a factorial mating design (Hodge et al., 1996). Subsequent studies using the trials established from this experiment showed significant positive genetic correlations between the additive genetic variation amongst the male parents with the open-pollinated female effects for traits under moderate to high additive genetic control, such as frost resistance (Volker et al., 1994), foliar disease damage (Dungey et al., 1997), wood density (Volker, 2002), the timing of the transition to adult foliage and first flowering (Jordan et al., 1999). However, this is not the case for growth (Hodge et al., 1996; Volker, 2002), a trait known to be under low additive genetic control in E. globulus (Costa e Silva et al., 2004; 2009; $\mathrm{Li}$ et al., 2007) and to exhibit severe inbreeding depression (Hardner and Potts, 1995; Hardner et al., 1998). This poor correlation is unlikely to be due to maternal effects (Lopez et al., 2003) and there is evidence in E. globulus (Hardner et al., 1996b), and other eucalypt species (Burgess et al., 1996; Hardner and Potts, 1997), that it may be partly explained by variation in outcrossing rates among parents. However, there is 
little understanding of the causal components of genetic variance for growth in inbred populations of E. globulus, and the extent to which variation in outcrossing rates is confounded with variation in non-additive genetic effects, including inbreeding depression, is unknown.

In the present study, we aim to better understand the genetic architecture of growth in E. globulus undergoing inbreeding. In particular, we attempt to provide an insight into the genetic basis of variation in inbreeding depression by looking at: (i) how open-pollinated progeny affects heritability estimation without proper accounting of inbreeding; (ii) whether inbreeding depression varies among parent trees; (iii) the extent to which family-level genetic effects in selfed and openpollinated populations are correlated with the additive genetic effects in a non-inbred population; (iv) the causal components of genetic variation under inbreeding.

\section{MATERIALS AND METHODS}

E. globulus families were generated from selfing (SELF), open pollination (OP) and unrelated polymix crossing (POL) of common parents from a native population in south-eastern Tasmania, and planted in a field trial. SELF had 27 and 26 seed parents in common with OP and POL, respectively, and there were 48 parents in common between OP and POL. Details about the plant material, crossing design and trial layout used in this study have been described previously (Costa e Silva et al., 2010).

A linear mixed model incorporating heterogeneity of (co)variances among (fixed) cross types for (random) family and residual effects was fitted to a data vector containing individual tree measures of breast-height diameter (DBH) at ages 4, 6 or 10 years from planting (Costa e Silva et al., 2010). Restricted maximum likelihood (REML) estimates of (co)variance components were obtained by using the average information REML algorithm, implemented in the ASReml software (Gilmour et al., 2006). Based on these observational variance components, narrow-sense heritability estimates $\left(\hat{h}^{2}\right)$ were obtained for the POL and OP progenies with the general formula:

$$
\hat{h}^{2}=\frac{1}{r}\left(\hat{\sigma}_{\mathrm{f}}^{2} / \hat{\sigma}_{\mathrm{p}}^{2}\right)
$$

where $r$ is the additive relationship coefficient, and $\hat{\sigma}_{\mathrm{f}}^{2}$ and $\hat{\sigma}_{\mathrm{p}}^{2}$ are the estimated between-family and phenotypic variances for a given cross type. In the first instance, unadjusted heritabilities of the POL $\left(\hat{h}_{\mathrm{pol}}^{2}\right)$ and OP $\left(\hat{h}_{\mathrm{op}}^{2}\right)$ progenies were estimated using $r=0.25$, assuming halfsib families and no inbreeding in the founder parents. Then, adjusted heritability of the POL progeny $\left(\hat{h}_{\text {pol(adj) }}^{2}\right)$ was estimated with $r=0.29$, calculated from $(1+f) / 4$, where $f=\beta /(2-\beta)$ is the equilibrium level of inbreeding for a population undergoing mixed selfing and random mating in proportions $\beta$ and $1-\beta$ (Cockerham and Weir, 1984), and assuming unrelated female parents and $\beta=0.3$. The $\hat{h}^{2}$ values from OP were also adjusted ( $\left.\hat{h}_{\mathrm{op}(\mathrm{adj})}^{2}\right)$ using $r=0.4$, calculated according to Squillace (1974) by assuming unrelated parents, $\beta=0.3$, five equally effective local males per female parent (and unrelated with the female parent) and no non-local pollen. An average outcrossing rate of 0.7 has been a common assumption in genetic analyses of E. globulus (Lopez et al., 2002), and thus the use of $\beta=0.3$ has become a standard by which to compare $\hat{h}^{2}$ values across studies. For a given cross type, $\hat{\sigma}_{\mathrm{p}}^{2}$ was calculated by summing $\hat{\sigma}_{\mathrm{f}}^{2}$, the estimated within-family variance and (when it was $>0$ ) the variance due to the interaction between replicates and families.

Estimates of family-level inbreeding depression $\left(F I D_{\mathrm{k}}\right)$ from selfing were obtained for DBH at each age as (see Moorad and Wade, 2005):

$$
F I D_{\mathrm{k}(\mathrm{self})}=\mathrm{LSM}_{(\mathrm{pol})}-\mathrm{LSM}_{(\mathrm{self})}+\hat{g}_{\mathrm{k}(\mathrm{pol})}-\hat{g}_{\mathrm{k}(\mathrm{self})}
$$

where $\operatorname{LSM}_{(\mathrm{pol})}-\mathrm{LSM}_{(\mathrm{self})}$ is the mean inbreeding depression and, for the POL and SELF crosses, $\mathrm{LSM}_{(\mathrm{pol})}$ and $\mathrm{LSM}_{(\text {self }}$ are estimated least-squares means, and $\hat{g}_{\mathrm{k}(\mathrm{pol})}$ and $\hat{g}_{\mathrm{k}(\mathrm{self})}$ pertain to effects of the $k$ th female parent. $F I D_{\mathrm{k}}$ estimates from open pollination $\left(F I D_{\mathrm{k}(\mathrm{op})}\right)$ were obtained by substituting $\operatorname{LSM}_{(\text {self })}$ with $\operatorname{LSM}_{(\mathrm{op})}$ and $\hat{g}_{\mathrm{k}(\mathrm{self})}$ with $\hat{g}_{\mathrm{k}(\mathrm{op})}$ in (2). Best linear unbiased predictors (BLUPs) of female parental effects under the different cross types were obtained by solving the mixed model equations. To compare $F I D_{\mathrm{k}}$ across ages, relative values of $F I D_{\mathrm{k}}$ were calculated according to Ågren and Schemske (1993) and Dudash et al. (1997). The variance among families in inbreeding depression $\left(\sigma_{\mathrm{FID}_{\mathrm{k}}}^{2}\right)$ from selfing was estimated at a given age as:

$$
\hat{\sigma}_{\mathrm{FID}(\mathrm{self})}^{2}=\hat{\sigma}_{\mathrm{f}(\mathrm{pol})}^{2}+\hat{\sigma}_{\mathrm{f}(\mathrm{self})}^{2}-2 \hat{\sigma}_{\mathrm{f}(\mathrm{pol}, \mathrm{self})}
$$

where $\hat{\sigma}_{\mathrm{f}(\mathrm{pol})}^{2}, \hat{\sigma}_{\mathrm{f}(\mathrm{self})}^{2}$ and $\hat{\sigma}_{\mathrm{f}(\mathrm{pol}, \mathrm{self})}$ are estimates of between-family variances for POL and SELF, and covariance between POL and SELF progenies related through a common female parent, respectively. Variation in family-level inbreeding depression with open pollination was also estimated $\left(\hat{\sigma}_{\mathrm{FID}(\mathrm{op})}^{2}\right)$, by replacing $\hat{\sigma}_{\mathrm{f}(\mathrm{self})}^{2}$ with $\hat{\sigma}_{\mathrm{f}(\mathrm{op})}^{2}$ and $\hat{\sigma}_{\mathrm{f}(\mathrm{pol}, \mathrm{self})}$ with $\hat{\sigma}_{\mathrm{f}(\mathrm{pol}, \mathrm{op})}$ in (3). The variation in family-level inbreeding depression is expected to be truly present when the variance amongst inbred progenies differs significantly from that amongst outbred families (Kelly, 2005). Thus, the $\hat{\sigma}_{\mathrm{FID}}^{2}$ estimation (and related $F I D_{\mathrm{k}(\mathrm{op})}$ values) was not pursued for age 10 , as $\hat{\sigma}_{\mathrm{f}(\mathrm{op})}^{2}$ was not significantly different from $\hat{\sigma}_{\mathrm{f}(\mathrm{pol})}^{2}$ for DBH at this age (Costa e Silva et al., 2010). Standard errors of the $\hat{\sigma}_{\mathrm{FID}_{\mathrm{k}}}^{2}$ were calculated as the square root of their sampling variances, obtained from the asymptotic matrix of sampling (co)variances of the REML estimates for the terms included in (3).

Correlations between cross types in family performance for DBH were calculated according to standard formulae (Lynch and Walsh, 1998), using REML estimates of between-family (co)variances. Onetailed likelihood ratio (LR) tests (Stram and Lee, 2004) were used to test whether the correlation estimated for a pair of cross types was significantly lower than one. Taylor series expansion was used to obtain approximate standard errors of the heritability and correlation estimates (Lynch and Walsh, 1998).

In a randomly mating population, the phenotypic variance of a trait can be defined as $V_{\mathrm{G}}+V_{\mathrm{E}}$, with $V_{\mathrm{G}}=V_{\mathrm{A}}+V_{\mathrm{D}}$ assuming no epistasis, where $V_{\mathrm{G}}, V_{\mathrm{A}}, V_{\mathrm{D}}$ and $V_{\mathrm{E}}$ are the total genetic, additive genetic, dominance and environmental variances, respectively. Inbreeding changes the population variance by introducing extra terms in $V_{\mathrm{G}}$, such as (Cockerham, 1983; Harris, 1964): $V_{\mathrm{DI}}$, the variance due to homozygous dominance deviations (i.e. dominance effects at loci where the allelic combinations are identical by descent); $C O V_{\mathrm{ADI}}$, the covariance between the additive effects of alleles and their homozygous dominance deviations; and $H^{*}$, the sum of squared inbreeding depression effects across loci. The crossing design in our study does not include full-sib family relationships and uses one level of controlled inbreeding only, and thus does not allow to separate the dominance parameters $V_{\mathrm{D}}, V_{\mathrm{DI}}$ and $H^{*}$. However, a simplification may be used to derive $V_{\mathrm{D}}$ and $V_{\mathrm{DI}}$ by assuming that all loci affecting the trait are biallelic, in which case $V_{\mathrm{D}}=H^{*}$, hence removing the need to estimate $H^{*}$ (Cockerham and Weir, 1984). The genetic expectations 
of the REML (co)variance estimates for DBH are (assuming linkage equilibrium, no epistasis and non-inbred founder parents):

$$
\begin{aligned}
\hat{\sigma}_{\mathrm{f}(\mathrm{pol})}^{2} & =1 / 4 V_{\mathrm{A}} \\
\hat{\sigma}_{\mathrm{f}(\text { pol,self })} & =1 / 2 V_{\mathrm{A}}+1 / 4 C O V_{\mathrm{ADI}} \\
\hat{\sigma}_{\mathrm{f}(\text { self })}^{2} & =V_{\mathrm{A}}+1 / 4 V_{\mathrm{D}}+C O V_{\mathrm{ADI}}+1 / 8 V_{\mathrm{DI}}
\end{aligned}
$$

and (assuming $V_{\mathrm{D}}=H^{*}$, as mentioned above),

$$
\begin{aligned}
\left(\hat{\sigma}_{\mathrm{f}(\mathrm{self})}^{2}+\hat{\sigma}_{\mathrm{wf}(\mathrm{self})}^{2}\right)-\left(\hat{\sigma}_{\mathrm{f}(\mathrm{pol})}^{2}+\hat{\sigma}_{\mathrm{wf}(\mathrm{pol})}^{2}\right) & \\
& =1 / 2 V_{\mathrm{A}}-1 / 4 V_{\mathrm{D}}+2 C O V_{\mathrm{ADI}}+1 / 2 V_{\mathrm{DI}}+V_{\mathrm{E}(\mathrm{self})}-V_{\mathrm{E}(\mathrm{pol})}
\end{aligned}
$$

where $\hat{\sigma}_{\mathrm{wf}(\mathrm{self})}^{2}$ and $\hat{\sigma}_{\mathrm{wf}(\mathrm{pol})}^{2}$ are within-family variance estimates, $V_{\mathrm{E}(\text { self })}$ and $V_{\mathrm{E}(\mathrm{pol})}$ are variances due to microenvironmental effects for SELF and POL, respectively. If $V_{\mathrm{E}(\mathrm{self})}=V_{\mathrm{E}(\mathrm{pol})}$, then the linear combination in (7) is a function of genetic effects only, which will allow deriving $V_{\mathrm{A}}, V_{\mathrm{D}}, C O V_{\mathrm{ADI}}$ and $V_{\mathrm{DI}}$ based on Equations (4) to (7). While it may be reasonable to assume that environmental effects for SELF and POL may be similar for DBH at age 4, this may not be the case at later ages due to the onset of canopy closure and the expected development of competition (e.g. Hardner and Potts, 1997). In addition, as differences in survival between SELF and POL were significant after age 4 (Costa e Silva et al., 2010), it is expected that progenies from these cross types will differ in levels of response to environmental effects after this earlier age. Hence, estimation of the genetic components of variation was restricted to age 4 .

\section{RESULTS}

Table I presents the $\hat{h}^{2}$ values for DBH in the OP and POL

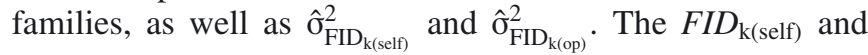
$F I D_{\mathrm{k}(\mathrm{op})}$ values for DBH are provided in Appendices ${ }^{1} 1$ and 2 , respectively; general combining ability (GCA) estimates of the female parents and related rankings are also shown in these appendices. Family correlations estimated between cross types are given in Table II.

The heritability estimates based on OP decreased gradually with age, whereas the opposite trend was observed for those from POL (Tab. I). At age 4, the $\hat{h}^{2}$ values from OP were $0.72 \pm 0.14$ before adjustment and $0.45 \pm 0.09$ after adjustment, compared with corresponding values of $\hat{h}_{\mathrm{pol}}^{2}=0.21 \pm 0.07$ and $\hat{h}_{\text {pol(adj) }}^{2}=0.18 \pm 0.06$ from POL. At age 6 , there was a fairly good agreement between $\hat{h}_{\mathrm{op}(\text { adj })}^{2}$ and both $\hat{h}_{\mathrm{pol}}^{2}$ and $\hat{h}_{\mathrm{pol}(\mathrm{adj})}^{2}$; in contrast, the unadjusted $\hat{h}_{\mathrm{op}}^{2}$ was almost twice as large (i.e. $0.50 \pm 0.12$ ). At age 10, the $\hat{h}^{2}$ values from OP were lower $\left(\hat{h}_{\mathrm{op}}^{2}=0.28 \pm 0.10\right.$ and $\left.\hat{h}_{\mathrm{op}(\mathrm{adj})}^{2}=0.18 \pm 0.06\right)$ than those from $\operatorname{POL}\left(\hat{h}_{\mathrm{pol}}^{2}=0.49 \pm 0.11\right.$ and $\left.\hat{h}_{\text {pol(adj) }}^{2}=0.42 \pm 0.10\right)$.

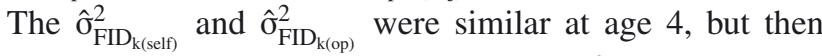

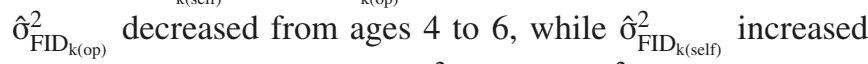

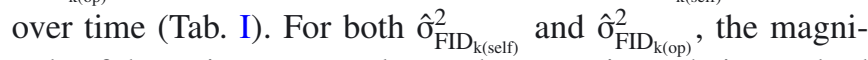
tude of the estimates were larger than two times their standard errors at all ages except for $\hat{\sigma}_{\mathrm{FID}_{\mathrm{k}(\mathrm{self})}}^{2}$ at age 10. The high mortality observed for SELF at 10 years (Costa e Silva et al., 2010)

\footnotetext{
${ }^{1}$ Appendices are available online only at www.afs-journal.org.
}

is likely to have affected the precision of $\hat{\sigma}_{\mathrm{FID}}^{2} \mathrm{D}_{\mathrm{kself}}$ at this age. Yet, in general, the $\hat{\sigma}_{\mathrm{FID}_{\mathrm{k}}}^{2}$ appear to differ significantly from zero, assuming asymptotic normality for the sampling distribution of the parameter and considering that an estimate larger than two standard errors is statistically significant at the 5\% level. $F I D_{\mathrm{k} \text { (self) }}$ ranged from 0.5 to $3.9 \mathrm{~cm}$ at age 4 , from 0.2 to $4.4 \mathrm{~cm}$ at age 6 , and from -1.0 to $5.3 \mathrm{~cm}$ at age 10 (Appendix ${ }^{1} 1$ ), indicating that the seed parents varied appreciably in their degree of inbreeding depression. For $F I D_{\mathrm{k}(\mathrm{op})}$, the ranges were -0.4 to $3.4 \mathrm{~cm}$ at age 4 , and -0.7 to $2.7 \mathrm{~cm}$ at age 6 , with most of the parents having reduced DBH growth in their OP offspring due to inbreeding (Appendix ${ }^{1} 2$ ). The relative values of $F I D_{\mathrm{k}(\mathrm{self})}$ and $F I D_{\mathrm{k}(\mathrm{op})}$ decreased with age for the majority of the parents, suggesting a gradual purging of inbreeding depression over time in the SELF and OP progenies. There was no evident relationship between $F I D_{\mathrm{k}}$ and predictions of parental breeding value for DBH: Pearson correlation coefficients with GCA ranged from -0.07 to 0.28 for $F I D_{\text {k(self), }}$, and from 0.17 to 0.22 for $F I D_{\mathrm{k}(\mathrm{op})}$; none of these small correlations were significantly different from zero at the $5 \%$ level. Conversely, Pearson correlation coefficients between $F I D_{\mathrm{k}(\text { self) }}$ and $F I D_{\mathrm{k}(\mathrm{op})}$ from common parents were 0.67 at age 4 , and 0.75 at age 6 , being both statistically significant $(P \leq 0.001)$. The relationship between $F I D_{\mathrm{k}(\text { self })}$ and $F I D_{\mathrm{k}(\mathrm{op})}$ from common parents is illustrated in Figure 1 for ages 4 and 6. Similar results were obtained with the relative values of $F I D_{\mathrm{k}(\text { self })}$ and $F I D_{\mathrm{k}(\mathrm{op})}$.

The family correlation estimates between cross types differed significantly $(P \leq 0.001)$ from one in all cases except for the pair SELF/OP at age 10 (Tab. II). Parental effects estimated from SELF tended to be highly correlated with corresponding effects from OP. Conversely, the POL families were in general poorly correlated with either SELF or OP progenies from the same parent. However, the correlation among parent effects from OP and POL improved over time: indeed, twotailed LR tests indicated that the correlation estimate among families from these cross types was significantly $(P \leq 0.05)$ different from zero at age 10, a result that was not detected at previous ages (not shown). For SELF and POL, none of the family correlation estimates differed significantly from zero $(P>0.05$, not shown $)$.

For DBH expressed under selfing, and using the relationships given in equations (4) to (7), the estimated genetic components of variation at age 4 were $\hat{V}_{\mathrm{A}}=0.96 \pm 0.32, \hat{V}_{\mathrm{D}}=$ $1.10 \pm 1.39, C \hat{O} V_{\mathrm{ADI}}=-1.24 \pm 0.82$ and $\hat{V}_{\mathrm{DI}}=9.71 \pm 3.52$, indicating that the variance due to homozygous dominance effects was noticeably larger than the estimates associated with other non-additive terms and additive effects, which appear to be similar. Estimates of components of genetic variation under inbreeding, derived from the resemblance among inbred and outbred relatives, are typically associated with large sampling variances unless prohibitively large sample sizes are used (Kelly, 1999). Nevertheless, for $\hat{V}_{\mathrm{A}}$ and $\hat{V}_{\mathrm{DI}}$, the magnitude of the estimates exceeded their standard errors by more than two times, suggesting that they differ significantly from zero. Conversely, for $\hat{V}_{\mathrm{D}}$ and $C \hat{O} V_{\mathrm{ADI}}$, the standard errors were 
Table I. Estimated narrow-sense heritabilities and variances in family-level inbreeding depression for DBH, based on progeny data from three crossing designs - selfing (SELF), open pollination (OP) and outcrossing with a polymix (POL) - at different ages from planting. The approximate standard errors of the parameter estimates are given within parenthesis.

\begin{tabular}{|c|c|c|c|c|c|c|}
\hline \multirow[t]{2}{*}{$\begin{array}{l}\text { Age } \\
\text { (years) }\end{array}$} & \multicolumn{4}{|c|}{ Narrow-sense heritabilities } & \multicolumn{2}{|c|}{$\begin{array}{l}\text { Variance in family-level } \\
\text { inbreeding depression }\end{array}$} \\
\hline & $\hat{h}_{\mathrm{op}}^{2}$ & $\hat{h}_{\mathrm{op}(\mathrm{adj})}^{2}$ & $\hat{h}_{\mathrm{pol}}^{2}$ & $\hat{h}_{\mathrm{pol}(\text { adj })}^{2}$ & $\hat{\sigma}_{\mathrm{FID}}^{2}($ self $)$ & $\hat{\sigma}_{\mathrm{FID}}^{2}(\mathrm{op})$ \\
\hline 4 & $\begin{array}{c}0.72 \\
( \pm 0.14)\end{array}$ & $\begin{array}{c}0.45 \\
( \pm 0.09)\end{array}$ & $\begin{array}{c}0.21 \\
( \pm 0.07)\end{array}$ & $\begin{array}{c}0.18 \\
( \pm 0.06)\end{array}$ & $\begin{array}{c}1.11 \\
( \pm 0.50)\end{array}$ & $\begin{array}{c}1.56 \\
( \pm 0.40)\end{array}$ \\
\hline 6 & $\begin{array}{c}0.50 \\
( \pm 0.12)\end{array}$ & $\begin{array}{c}0.31 \\
( \pm 0.07)\end{array}$ & $\begin{array}{c}0.33 \\
( \pm 0.09)\end{array}$ & $\begin{array}{c}0.28 \\
( \pm 0.07)\end{array}$ & $\begin{array}{c}2.88 \\
( \pm 1.19)\end{array}$ & $\begin{array}{c}1.29 \\
( \pm 0.38)\end{array}$ \\
\hline 10 & $\begin{array}{c}0.28 \\
( \pm 0.10)\end{array}$ & $\begin{array}{c}0.18 \\
( \pm 0.06)\end{array}$ & $\begin{array}{c}0.49 \\
( \pm 0.11)\end{array}$ & $\begin{array}{c}0.42 \\
( \pm 0.10)\end{array}$ & $\begin{array}{c}6.36 \\
( \pm 3.34)\end{array}$ & $\mathrm{NE}$ \\
\hline
\end{tabular}

$\hat{h}_{\mathrm{op}}^{2}$ and $\hat{h}_{\mathrm{op}(\mathrm{adj})}^{2}=$ unadjusted and adjusted heritability estimates from the OP progeny; $\hat{h}_{\mathrm{pol}}^{2}$ and $\hat{h}_{\mathrm{pol}(\mathrm{adj})}^{2}$ unadjusted and adjusted heritability estimates from the POL progeny; $\hat{\sigma}_{\mathrm{FID}_{\mathrm{k}}(\mathrm{self})}^{2}$ and $\hat{\sigma}_{\mathrm{FID}}^{2}(\mathrm{op})=$ estimated variances in family-level inbreeding depression due to selfing and open pollination; NE = not estimated, as estimates of OP and POL family variances were not significantly $(P>0.05)$ different.

Table II. Estimated family correlations (with their approximate standard errors given within parenthesis) between different crossing designs for DBH at different ages from planting. The significance probabilities refer to one-tailed likelihood ratio tests applied to test the departure of the correlation estimates from one.

\begin{tabular}{|c|c|c|c|c|c|c|}
\hline & \multicolumn{2}{|c|}{4 years } & \multicolumn{2}{|c|}{6 years } & \multicolumn{2}{|c|}{10 years } \\
\hline & SELF & $\overline{\mathrm{OP}}$ & SELF & OP & SELF & OP \\
\hline OP & $\begin{array}{c}0.59 \\
( \pm 0.19) \\
P \leq 0.001\end{array}$ & & $\begin{array}{c}0.64 \\
( \pm 0.19) \\
P \leq 0.001\end{array}$ & & $\begin{array}{c}0.99 \\
( \pm 0.17) \\
P>0.05\end{array}$ & \\
\hline POL & $\begin{array}{c}0.31 \\
( \pm 0.29) \\
P \leq 0.001\end{array}$ & $\begin{array}{c}0.14 \\
( \pm 0.20) \\
P \leq 0.001\end{array}$ & $\begin{array}{c}0.10 \\
( \pm 0.26) \\
P \leq 0.001\end{array}$ & $\begin{array}{c}0.31 \\
( \pm 0.18) \\
P \leq 0.001\end{array}$ & $\begin{array}{c}0.39 \\
( \pm 0.26) \\
P \leq 0.001\end{array}$ & $\begin{array}{c}0.45 \\
( \pm 0.18) \\
P \leq 0.001\end{array}$ \\
\hline
\end{tabular}

The crossing designs are selfing (SELF), open pollination (OP) and outcrossing with a polymix (POL).

A

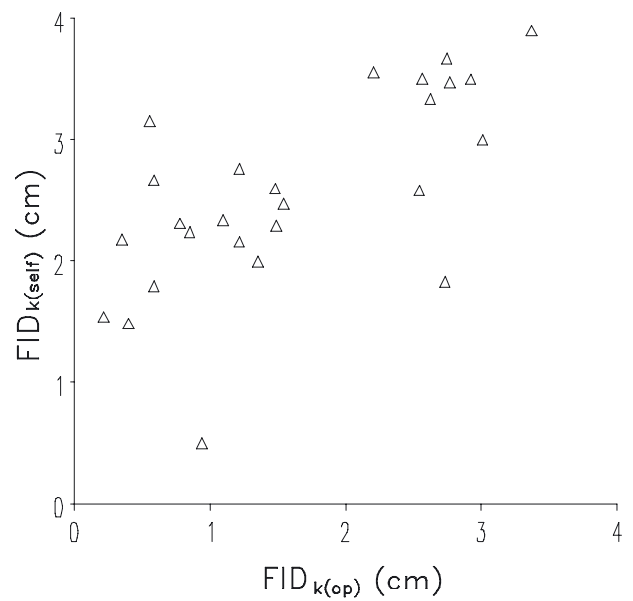

B

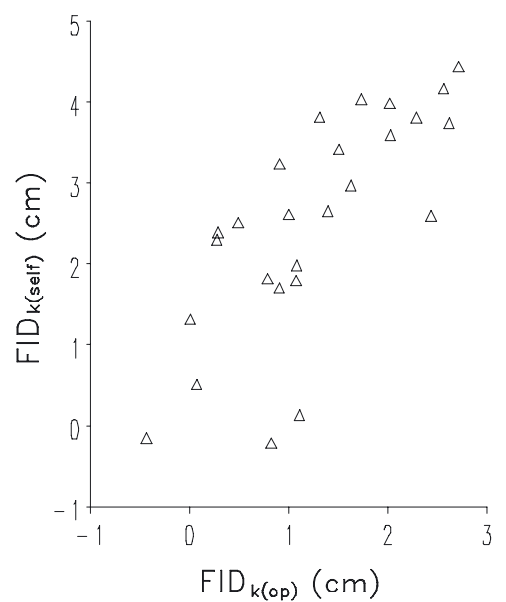

Figure 1. Relationship between estimates of family-level inbreeding depression from selfing $\left(F I D_{\mathrm{k}(\text { self })}\right)$ and open pollination $\left(F I D_{\mathrm{k}(\mathrm{op})}\right)$ from common parents at ages 4 (A) and $6(\mathrm{~B})$ years from planting. Pearson correlation coefficients between $F I D_{\mathrm{k}(\text { self })}$ and $F I D_{\mathrm{k}(\mathrm{op})}$ were 0.67 at age 4 , and 0.75 at age 6 , being both statistically significant $(P \leq 0.001)$. 
large compared with the magnitude of the estimates, and thus these could be the result of sampling error.

\section{DISCUSSION}

\subsection{Narrow-sense heritabilities}

For growth traits in Eucalyptus spp., overestimation of heritabilities based on OP progeny relative to estimates derived from controlled crosses may be due to several causes, such as mixture of inbred and outbred individuals within families, population-level inbreeding depression, and heterogeneous self-pollination rates and/or inbreeding depression amongst families (Borralho, 1994; Hodge et al., 1996). At ages 4 and 6 , the unadjusted $\hat{h}_{\text {op }}^{2}$ appeared to be noticeably biased upwards (Tab. I), indicating that the assumption of a half-sib family structure $(r=0.25)$ for the OP offspring was not valid at these ages. Squillace's (1974) correction of the mean coefficient of additive relationship to adjust $\hat{h}_{\text {op }}^{2}$ applies an average self-pollination rate assumed for the base population, but does not consider the population-level inbreeding depression and the differential inbreeding depression that may occur among OP families. Using $r=0.4$ was not sufficient to remove the upward bias in $\hat{h}_{\text {op }}^{2}$ at age 4 . Even when assuming a high value such as $\beta=0.5$, the use of Squillace's (1974) method could not correct for the overestimation in $\hat{h}_{\text {op }}^{2}$ at age 4 (not shown). Indeed, at age 4, inbreeding depression in the OP population had its highest magnitude (i.e. 10\%) for $\mathrm{DBH}$, and $\hat{\sigma}_{\mathrm{f}(\mathrm{op})}^{2}$ had its greatest level of inflation relative to $\hat{\sigma}_{\mathrm{f}(\mathrm{pol})}^{2}$ (Costa e Silva et al., 2010). The later suggests a larger differential inbreeding depression among OP families at age 4, consistent with the greater $\hat{\sigma}_{\mathrm{FID}}^{2}$ value presented in Table I. Yet, these events were attenuated at age 6, in which case Squillace's (1974) procedure was effective for reducing the bias in the $\hat{h}_{\mathrm{op}}^{2}$.

\subsection{Variation in family-level inbreeding depression}

The inflation of $\hat{\sigma}_{\mathrm{f}}^{2}$ in SELF or OP relative to POL, as reported in Costa e Silva et al. (2010), reflects the heterogeneity in inbreeding depression amongst the SELF or OP families, as quantified by $\hat{\sigma}_{\mathrm{FID}_{\mathrm{k}}}^{2}$ (Tab. I). Assuming a model based on rare recessive deleterious alleles, Moorad and Wade (2005) showed that low values of the average degree of dominance $(h)$, referring to increased dominance deviations in heterozygotes and increased mean inbreeding depression, provided more variation in family-level inbreeding depression than did high values of $h$. In particular, for partially recessive alleles with $h=0.2$, $\sigma_{\mathrm{FID}_{\mathrm{k}}}^{2} \approx 1.6 V_{\mathrm{A}}$ after one generation of selfing (see Eq. (8) of Moorad and Wade, 2005); this approaches $\hat{\sigma}_{\mathrm{FID}}^{2} \approx 1.2 \hat{V}_{\mathrm{A}(\mathrm{self})}$ and $\hat{\sigma}_{\mathrm{FID}(\mathrm{pop})}^{2} \approx 1.6 \hat{V}_{\mathrm{A}}$ that can be estimated for $\mathrm{DBH}$ at age 4 in the present study. These results suggest that the expression of deleterious alleles that are rare and partially recessive is likely to be a major source of inbreeding depression in DBH. There was also a prevailing pattern amongst female parents for a decrease in relative $F I D_{\mathrm{k}}$ values with age (Appendices ${ }^{1} 1$ and 2 ).
This indicates that, through purging of partially deleterious recessive alleles, dominance rather than overdominance underlies the expression of inbreeding depression (Dudash et al., 1997), although avoiding competition among cross types in the field trial may have also contributed to reduce the differences between SELF (or OP) and POL at a later age (Costa e Silva et al., 2010). With lower $h$, the contribution of additive genetic effects to $\sigma_{\mathrm{FID}_{\mathrm{k}}}^{2}$ will gradually be reduced and, with complete dominance (i.e. $h=0$ ), nearly all $\sigma_{\mathrm{FID}_{\mathrm{k}}}^{2}$ will be generated by non-additive genetic variation (in which case $\sigma_{\mathrm{FID}_{\mathrm{k}}}^{2}$ is much greater than $V_{\mathrm{A}}$; Moorad and Wade, 2005).

Comparable $\hat{\sigma}_{\mathrm{FID}_{k}}^{2}$ in the SELF and OP populations at age 4 (Tab. I), coupled with a positive correlation between the $F I D_{\mathrm{k}(\text { self })}$ and $F I D_{\mathrm{k}(\mathrm{op})}$ of common parents (Fig. 1), argues that the variation in non-additive effects arising from inbreeding per se (i.e. rather than variation in outcrossing rate) may be the main driver of the variation in performance amongst OP families in early growth. From a deployment perspective, it is clear that unless outcrossing rates are high in open-pollinated seed orchards, the severe effects of inbreeding depression may counter any additive genetic gains in growth made through parental selection. This risk has led to the development of systems for mass production of control-pollinated seed of E. globulus (Potts et al., 2008). These systems have the potential to exploit non-additive genetic effects of both random and inbred origin. However, given the deleterious effects of inbreeding in E. globulus and the magnitude of the variance in family-level inbreeding depression relative to the additive genetic variance (as indicated above), the major initial gain is likely to be from the avoidance of inbreeding arising from the species' mixed mating system.

\subsection{Family correlations between cross types}

The performance of selfed families could not provide a reliable indicator of the parental GCA for $\mathrm{DBH}$, as indicated by the poor family correlation estimates between the SELF and POL cross types (Tab. II). This corroborates the mode of gene action suggested from the magnitude of the $\hat{\sigma}_{\mathrm{f}(\text { self })}^{2} / \hat{\sigma}_{\mathrm{f}(\mathrm{pol})}^{2}$ ratios (Costa e Silva et al., 2010) and $\hat{\sigma}_{\mathrm{FID}_{\mathrm{k}(\mathrm{self})}^{2}}$ values (Tab. I), in that correlations among family effects from selfed and outcrossed progenies are expected to be low if non-additive genetic variance contributes substantially to genetic variation under inbreeding. Thus, during the initial stages of domestication, the use of selfs for progeny testing may not be an adequate strategy for selecting superior parents for outcross growth in E. globulus.

Parental effects estimated from the OP offspring were in general well correlated with the selfed performance, and thus tended to be also poor indicators of GCA. Strategies to raise the accuracy of parental effects obtained from OP progeny include accounting for native stand structure (Borralho and Potts, 1996), only sampling seed at high levels in the tree canopies (where outcrossing rates are greater and more homogeneous, Patterson et al., 2001), as well as a direct estimation of outcrossing rates (Burgess et al., 1996). However, these 
strategies cannot account for the inherent variation in the genetic factors giving rise to inbreeding depression which vary between OP families of E. globulus. Nevertheless, the present study indicated that the correlation between a parent's OP and POL progeny performance increased over time (Tab. II), suggesting that deleterious alleles may have been purged in the OP progeny during the course of the experiment, which is supported at age 10 by an insignificant population-level inbreeding depression due to open pollination and by similar $\hat{\sigma}_{\mathrm{f}(\mathrm{op})}^{2}$ and $\hat{\sigma}_{\mathrm{f}(\mathrm{pol})}^{2}$ values (Costa e Silva et al., 2010). Thus, although it may not be effective at earlier ages, open pollination may become a useful method for progeny testing for growth as field trials get older and OP family effects turn to be more reliable predictors of parental breeding values. Yet, this may be achieved at the expense of a reduction in genetic gain per unit of time, which may limit the use of family selection based on OP progeny as a breeding tool in E. globulus. This limitation does not appear to apply to the non-growth traits studied to date (see Introduction).

\subsection{Genetic variation under inbreeding}

The combined effects of dominance and inbreeding lead to the inclusion of five genetic components in the covariance between self relatives. Of the three genetic components $-V_{\mathrm{DI}}$, $C O V_{\mathrm{ADI}}$ and $H^{*}$ - that are a direct consequence of inbreeding, only $C O V_{\mathrm{ADI}}$ can be negative depending on the gene frequencies and dominance effects (Cockerham, 1983; Cockerham and Weir, 1984). The present study indicated that $\hat{V}_{\text {DI }}$ had a major contribution to the estimated genetic variation under inbreeding in this population. In particular, the much smaller contribution of $\hat{V}_{\mathrm{D}}$ compared to $\hat{V}_{\mathrm{DI}}$ appears despite the significant $(P \leq 0.001)$ and high (i.e. $21 \%)$ population-level inbreeding depression due to selfing that was detected for DBH at age 4 (Costa e Silva et al., 2010). Yet, while apparently not significant, $\hat{V}_{\mathrm{D}}$ was comparable to $\hat{V}_{\mathrm{A}}$, consistent with results obtained in growth traits by Li et al. (2007) for non-inbred populations of E. globulus. Results from other studies with quantitative traits have indicated that significant inbreeding depression was generally accompanied by an important contribution of estimated $V_{\mathrm{DI}}$ to genetic variation, but not always by significant estimates of $V_{\mathrm{D}}$ and/or $C O V_{\mathrm{ADI}}$ (Kelly and Arathi, 2003; Shaw et al., 1998). The significant inbreeding depression due to selfing together with the substantial $\hat{V}_{\text {DI }}$, as found here for $\mathrm{DBH}$ at age 4 , indicate that the homozygous dominance effects may be strongly directional at loci influencing the trait, so that inbreeding increases genetic variance while decreasing significantly the trait mean.

Results reported by Moorad and Wade (2005) indicated that $V_{\text {DI }}$ may be a major contributor to variation in inbreeding depression amongst selfed families. In particular, for partial dominance with rare alleles, $V_{\mathrm{DI}} \approx 11 V_{A}$ and $V_{\mathrm{DI}} \approx 5 V_{\mathrm{A}}$ for average degrees of dominance $(h)$ of 0.15 and 0.20 , respectively (see Eq. (7), Moorad and Wade, 2005). This range of values includes $\hat{V}_{\mathrm{DI}} \approx 10 \hat{V}_{\mathrm{A}}$, which can be calculated from the genetic components estimated for DBH at age 4 , and thus giving another indication that deleterious alleles that are rare and partially recessive are likely to be important in inducing inbreeding depression for this trait. In addition, for $h$ values varying from 0.15 to 0.20 , rare alleles may make a substantial greater contribution to $V_{\mathrm{DI}}$ than to $C O V_{\mathrm{ADI}}$, as suggested from the work of Moorad and Wade (2005). Negative $C O V_{\mathrm{ADI}}$ may appear when partially recessive deleterious alleles with frequencies similar to or greater than 0.5 are also contributing to inbreeding depression in a trait (Kelly, 1999; Resende and Vencovsky, 1992). This may be also the case for DBH at age 4 , as suggested by the negative sign of $C \hat{O} V_{\mathrm{ADI}}$. Yet, given its poor precision, the estimate obtained here for $C O V_{\mathrm{ADI}}$ may be due to sampling error, and thus we cannot exclude the possibility that the actual value of this parameter is close to zero.

The magnitudes of $V_{\mathrm{DI}}$ and $C O V_{\mathrm{ADI}}$ are expected to affect the response to selection for improving a trait in a population undergoing inbreeding, as they may aggravate the depressing effect of inbreeding on the trait mean. In particular, if $C O V_{\mathrm{ADI}}$ is negative, then the higher the additive effect of an allele, the higher will be its contribution to inbreeding depression caused by homozygous dominance deviations. In this sense, in a population undergoing inbreeding, selection towards the increase of the frequency of high-valued alleles could also increase inbreeding depression in the next generations, and thus delay genetic progress. This could offset the potential benefits of strategies using selfing as a breeding tool, which may enable rare recessive alleles with major deleterious effects to be eliminated in early generations of breeding (Williams and Savolainen, 1996). For populations undergoing mixed mating, Shaw et al. (1998) reported that, together with a large and significant estimate for $V_{\mathrm{DI}}$, even small, negative estimates of $C O V_{\mathrm{ADI}}$ reduced the expected response to selection much more than could be accounted for by inbreeding depression alone; the reduction was somewhat greater for higher selfing rates. Thus, for E. globulus OP populations, the impact of $C O V_{\mathrm{ADI}}$ and/or $V_{\mathrm{DI}}$ on selection response may be appreciable given the moderate values found for average outcrossing rates under open pollination (Mimura et al., 2009). Resende and Vencovsky (1992) also reported that the advantage of selection based on progeny testing of selfed families (after one generation of selfing) over half-sibs increased as populations became more improved, which was due to the negative $C O V_{\mathrm{ADI}}$ at intermediate to low frequencies of the favourable alleles.

\section{CONCLUSION}

OP heritability estimates for $\mathrm{DBH}$ growth decreased with time, while an opposite trend was observed in the POL population, indicating that the presence of inbreds and associated non-additive genetic variance in the OP population may have biased upwards the estimated OP heritability at an early age.

There was virtually no correlation between selfed families and additive genetic effects of the seed parent for DBH growth, indicating the presence of large non-additive genetic effects due to inbreeding. In addition, this study showed that estimates of parental effects for DBH from OP progenies, particularly those derived from native stand seed collections, are likely to be of poor accuracy and full pedigree control will be 
required for their accurate estimation. Nevertheless, our results also suggested that, after allowing for the mortality of inbred individuals in the $\mathrm{OP}$ offspring, it is possible that later age trial measurements may better correlated to the additive genetic effects in the population.

An estimated component of genetic variance due to dominance effects resulting from inbreeding appeared to be a major contributor to the variation in inbreeding depression amongst selfed families. Indeed, the estimated variance due to homozygous dominance deviations for $\mathrm{DBH}$ growth under selfing was nearly 10 -fold greater than the dominance variance associated with random mating and the additive variance. The importance of this dominance variance due to inbreeding may reflect the build up of a genetic load of rare deleterious alleles in the central parts of the species range.

Acknowledgements: We thank Gunns Ltd for provision of the trial site, Paul Tilyard for data collection and management. Funding for crossing and trial establishment was provided by the CRC for Temperate Hardwood Forestry. Trial measurements were supported by the CRC for Sustainable Production Forestry and CRC for Forestry. The financial support given by Fundação para a Ciência e Tecnologia (Lisboa, Portugal) through the Ciência 2007 initiative to the senior author and by the Australian Research Council (BMP) is gratefully acknowledged.

\section{REFERENCES}

Ågren J. and Schemske D.W., 1993. Outcrossing rate and inbreeding depression in two annual monoecious herbs, Begonia hirsuta and $B$. semiovata. Evolution 47: 125-135.

Borralho N.M.G., 1994. Heterogeneous selfing rates and dominance effects in estimating heritabilities from open-pollinated progeny. Can. J. For. Res. 24: 1079-1082.

Borralho N.M.G. and Potts B.M., 1996. Accounting for native stand characteristics in genetic evaluations of open-pollinated progeny from Eucalyptus globulus base population. New For. 11: 53-64.

Burgess I.P., Williams E.R., Bell J.C., Harwood C.E., and Owen J.V., 1996. The effect of outcrossing rate on the growth of selected families of Eucalyptus grandis. Silvae Genet. 45: 97-101.

Cockerham C.C., 1983. Covariances of relatives from self-fertilization. Crop Sci. 23: 1177-1180.

Cockerham C.C. and Weir B.S., 1984. Covariances of relatives stemming from a population undergoing mixed self and random mating. Biometrics 40: 157-164.

Costa e Silva J., Borralho N.M.G., and Potts B.M., 2004. Additive and non-additive genetic parameters from clonally replicated and seedling progenies of Eucalyptus globulus. Theor. Appl. Genet. 108: 1113-1119.

Costa e Silva J., Borralho N.M.G., Araújo J.A., Vaillancourt R.E., and Potts B.M., 2009. Genetic parameters for growth, wood density and pulp yield in Eucalyptus globulus. Tree Genet. Genomes 5: 291-305.

Costa e Silva J., Hardner C.M., Tilyard P., Pires A.M., and Potts B.M., 2010. Effects of inbreeding on population mean performance and observational variances in Eucalyptus globulus, Ann. For. Sci. 67: 605.

Dudash M.R., Carr D.E., and Fenster C.B., 1997. Five generations of enforced selfing and outcrossing in Mimulus guttatus. Inbreeding depression variation at the population and family level. Evolution 51: $54-65$.
Dungey H.S., Potts B.M., Carnegie A.J., and Ades P.K., 1997. Mycosphaerella leaf disease: Genetic variation in damage to Eucalyptus nitens, E. globulus and their $\mathrm{F}_{1}$ hybrid. Can. J. For. Res. 27: 750-759.

Edwards J.W. and Lamkey K.R., 2002. Quantitative Genetics of Inbreeding in a synthetic maize population. Crop Sci. 42: 1094-1104.

Eldridge K., Davidson J., Harwood C., and van Wyk G., 1993. Eucalypt domestication and breeding. Clarendon Press, Oxford, UK.

Gilmour A.R., Gogel B.J., Cullis B.R., and Thompson R., 2006. ASReml User Guide Release 2.0. VSN International Ltd, Hemel Hempstead, UK.

Hardner C.M. and Potts B.M., 1995. Inbreeding depression and changes in variation after selfing Eucalyptus globulus subsp. globulus. Silvae Genet. 44: 46-54.

Hardner C.M. and Potts B.M., 1997. Postdispersal selection following mixed mating in Eucalyptus regnans. Evolution 51: 103-111.

Hardner C.M., Borralho N.M.G., Tier B., Miller S., and Goddard M., 1996a. Accounting for dominance and inbreeding in genetic evaluations using individual tree mixed models. In: Dieters M.J., Matheson A.C., Nikles D.G., Harwood C.E., and Walker S.M. (Eds.), Tree Improvement for Sustainable Tropical Forestry, Proceedings QFRI-IUFRO Conference, 27 October - 1 November 1996, Caloundra, Queensland, Australia. Queensland Forest Research Institute, Caloundra, Queensland, pp. 143-147.

Hardner C.M., Vaillancourt R.E., and Potts B.M., 1996b. Stand density influences outcrossing rate and growth of open-pollinated families of Eucalyptus globulus. Silvae Genet. 45: 226-228.

Hardner C.M., Potts B.M., and Gore P.L., 1998. The relationship between cross success and spatial proximity of Eucalyptus globulus ssp. globulus parents. Evolution 52: 614-618.

Harris D.L., 1964. Genotypic covariances between inbred relatives. Genetics 50: 1319-1348.

Hodge G.R., Volker P.W., Potts B.M., and Owen J.V., 1996. A comparison of genetic information from open-pollinated and control-pollinated progeny tests in two eucalypt species. Theor. Appl. Genet. 92: 5363.

Jordan G., Potts B.M., and Wiltshire R., 1999. Strong, independent quantitative genetic control of vegetative phase change and first flowering in Eucalyptus globulus ssp. globulus. Heredity 83: 179-187.

Kelly J.K., 1999. An experimental method for evaluating the contribution of deleterious mutations to quantitative trait variation. Genet. Res. 73: $263-273$.

Kelly J.K., 2005. Family level inbreeding depression and the evolution of plant systems. New Phytol. 165: 55-62.

Kelly J.K. and Arathi H.S., 2003. Inbreeding and the genetic variance of floral traits in Mimulus guttatus. Heredity 90: 77-83.

Li Y., Dutkowski G.W., Apiolaza L.A., Pilbeam D.J., Costa e Silva J., and Potts B.M., 2007. The genetic architecture of a Eucalyptus globulus full-sib breeding population in Australia. For. Genet. 12: 167-179.

Lopez G.A., Potts B.M., Dutkowski G.W., Apiolaza L.A., and Gelid P.E., 2002. Genetic variation and inter-trait correlations in Eucalyptus globulus base population trials in Argentina. For. Genet. 9: 217-231.

Lopez G.A., Potts B.M., Vaillancourt R.E., and Apiolaza L.A., 2003. Maternal and carryover effects on early growth of Eucalyptus globulus. Can. J. For. Res. 33: 2108-2115.

Lynch M. and Walsh B., 1998. Genetics and Analysis of Quantitative Traits, Sinauer Associates, Sunderland, MA, USA.

Mimura M., Barbour R.C., Potts B.M., Vaillancourt R.E., and Watanabe K.N., 2009. Comparison of contemporary mating patterns in 
continuous and fragmented Eucalyptus globulus native forests. Mol. Ecol. 18: 4180-4192.

Moorad J.A. and Wade M.J., 2005. A genetic interpretation of the variation in inbreeding depression. Genetics 170: 1373-1384.

Patterson B., Vaillancourt R.E., and Potts B.M., 2001. Eucalypt seed collectors: beware of sampling seed lots from low in the canopy! Aust. For. 64: 139-142.

Potts B.M., McGowen M.H., Williams D.R., Suitor S., Jones T.H., Gore P.L., and Vaillancourt R.E., 2008. Advances in reproductive biology and seed production systems of Eucalyptus: The case of Eucalyptus globulus. Southern Forests 70: 145-154.

Resende M.D.V. and Vencovsky R., 1992. Numerical evaluation of the relative efficiency of Eucalyptus selection methods based on half-sib and selfed families. I. Progeny testing. In: Lambeth C.C. and Dvorak W. (Eds.), Resolving Tropical Forest Resource Concerns Through Tree Improvement, Gene Conservation and Domestication of New
Species, Proceedings IUFRO Conference, Group S2.02-08, Breeding Tropical Trees, Cartagena and Cali, Colombia, pp. 261-269.

Shaw R.G., Byers D.L., and Shaw F.H., 1998. Genetic components of variation in Nemophila menziesii undergoing inbreeding: morphology and flowering time. Genetics 150: 1649-1661.

Squillace A.E., 1974. Average genetic correlations among offspring from open-pollinated forest trees. Silvae Genet. 23: 149-156.

Stram D.O. and Lee J.W., 1994. Variance components testing in the longitudinal mixed effects model. Biometrics 50: 1171-1177.

Volker P.W., 2002. Quantitative Genetics of Eucalyptus globulus, E. nitens and their $\mathrm{F}_{1}$ Hybrid. Unpublished Ph.D. thesis, School of Plant Science, University of Tasmania, Hobart.

Volker P.W., Owen J.V., and Borralho N.M.G., 1994. Genetic variances and covariances for frost tolerance in Eucalyptus globulus and $E$. nitens. Silvae Genet. 43: 366-372.

Williams C.G. and Savolainen O., 1996. Inbreeding depression in conifers: implications for breeding strategy. For. Sci. 42: 102-117. 